\title{
Gestärkte Position des US-Präsidenten durch das Line-Item Veto. Erfahrungen aus der zweiten Clinton-Administration
}

\author{
Alexander Kühne
}

Das Regierungssystem der USA wird nach der Typologie der Regierungsformen bekanntermaßen dem präsidentiellen zugeordnet. ${ }^{1}$ Die Beziehungen zwischen Kongress und Präsident können dabei sehr unterschiedlich ausgeprägt sein und gleichermaßen zu einer machtvollen Präsidialregierung (Presidential Government), kulminierend in einer imperialen Präsidentschaft (Imperial Presidency) ${ }^{2}$, wie zu einer Vorherrschaft des Kongresses (Congressional Government $)^{3}$ führen. Die Verfassungsväter wollten einen wirksamen, aber keinen überragend starken Präsidenten; die Legislative sollte die Vorhand besitzen, wie es James Madison zum Ausdruck bringt: „In republican government the legislative authority necessarily predominates. "“ Die Verteilung der Macht zwischen Legislative, Exekutive und Judikative findet sich in der US-amerikanischen Verfassung in exakt dieser Reihenfolge. Das tatsächliche Verhältnis der Gewalten zueinander wird in der Politikwissenschaft gemeinhin auf die von Richard E. Neustadt geprägte Formel „separated institutions sharing powers"5 gebracht, die auf die funktionale Verschränkung beider Institutionen im politischen Prozess verweist. Dieser Standardlehrsatz der modernen amerikanischen Gewaltenteilungslehre wurde in späteren Arbeiten weiter ausdifferenziert. So charakterisiert Charles O. Jones die amerikanische Variante der Gewaltenteilung als „a government of separated institutions

1 Es gibt verschiedene Methoden demokratische Systeme zu typologisieren. Die Unterscheidung zwischen parlamentarischen und präsidentiellen Regierungssystemen beruht auf dem Kriterium der Machtverteilung zwischen Exekutive und Legislative. Vgl. dazu Winfried Steffani, Parlamentarische und präsidentielle Demokratie. Strukturelle Aspekte westlicher Demokratien, Opladen 1979; ders., Zur Unterscheidung parlamentarischer und präsidentieller Regierungssysteme, in: ZParl, 14. Jg. (1983), H. 3, S. 390 - 401; Arend Lijphart (Hrsg.), Parliamentary versus Presidential Government, Oxford 1992; auch James Bryce, Modern Democracies, Toronto 1921; Ernst Fraenkel, Parlamentarisches Regierungssystem, in: ders. / Karl Dietrich Bracher (Hrsg.), Staat und Politik, Frankfurt am Main 1957, S. 238 - 243; Karl Loewenstein, Verfassungslehre, Tübingen 1959; Douglas V. Verney, Analysis of Political Systems, in: Harry Eckstein / David E. Apter (Hrsg.), Comparative Politics. A Reader, New York 1966, S. 175 - 191; Matthew Soberg Shugart / John M. Carey, Presidents and Assemblies. Constitutional Design and Electoral Dynamics, Cambridge 1992; Robert Schulz, Neuere Ansätze zur Typologisierung demokratischer Systeme, Rostock 2003.

2 Vgl. Arthur M. Schlesinger, Jr., The Imperial Presidency, Boston 1973; Donald R. Wolfensberger, The Return of the Imperial Presidency?, in: Wilson Quarterly, 26. Jg. (2002), H. 1, S. 39; Andrew Rudalevige, The New Imperial Presidency. Renewing Presidential Power after Watergate, Ann Arbor 2005.

3 Den Vorrang des Kongresses beschrieb Woodrow Wilson 1885 als ,a government by the Standing Committees of Congress"; Woodrow Wilson, Congressional Government: A Study in American Politics, Baltimore 1956, S. 56. Vgl. auch Theodore J.Lowi / Benjamin Ginsberg / Kenneth A. Shepsle, American Government - Power and Purpose, New York / London 2004, S. 60.

4 James Madison, Federalist No. 51, in: Alexander Hamilton / ders. / John Jay, The Federalist - A Commentary on the Constitution of the United States, New York 2001, S. 332.

5 Richard E. Neustadt, Presidential Power, New York 1960, S. 33. 
competing for shared powers“6. Sergio Fabbrini spricht von einem „system of separated government with limited presidential pre-eminence "7, was dazu führt, das amerikanische Regierungssystem als ein „Parliamentary Presidentialism"8 zu bezeichnen.

Veränderungen des amerikanischen Gewaltenteilungssystems sind nicht selten auf Entscheidungen des Obersten Gerichtshofs zurückzuführen ${ }^{9}$, so auch im Falle des selektiven Vetos des Präsidenten im Haushaltsverfahren (Line-Item Veto) ${ }^{10}$, das im Weiteren ausführlicher diskutiert werden soll. Dazu werden die ersten beiden Jahre der zweiten Amtszeit William Jefferson Clintons (1997/1998) näher betrachtet, in denen der Demokratische Präsident über die zusätzliche Kompetenz verfügte, sein Veto nicht mehr nur gegen komplette Gesetze einzulegen, sondern auch einzelne Passagen aus Haushaltsgesetzen zu streichen. Insbesondere die Republikanische Mehrheit im Kongress, aber auch einige Demokraten verbanden mit dem Gesetz die Hoffnung auf Haushaltskonsolidierung. ${ }^{11} \mathrm{Ob}$ diese Hoffnung sich erfüllte oder das Line-Item Veto nicht noch ganz andere, systemische Konsequenzen für das Verhältnis von Exekutive und Legislative hatte, wird im Folgenden zu prüfen sein. Dass dies keineswegs nur von historiographischem Interesse ist, belegt der Blick auf die Präsidentschaft George W. Bushs, den Vorwahlkampf der Republikanischen Präsidentschaftskandidaten 2008 sowie jüngste Bestrebungen beider Parteien im Senat zur Senkung der Staatsausgaben. So forderte Bush in seinen beiden Amtszeiten den Kongress wiederholt auf, ihn mit einem selektiven Veto auszustatten ${ }^{12}$, und auch die Republikanischen Bewerber für die Präsidentschaftswahl 2008 unterstrichen im Vorwahlkampf ihre Ambitionen, das Line-Item Veto wieder einzuführen. ${ }^{13}$ Einen konkreten Vorstoß unternahmen mittlerweile die Senatoren John McCain (R, Arizona) und Russ Feingold (D, Wisconsin) sowie der Abgeordnete Paul Ryan (R, Wisconsin). Sie einigten sich am 4. März 2009 auf eine gemeinsame Gesetzesinitiative zur Wiedereinführung des selektiven Vetos („Congressional Accountability and Line-Item Veto Act of 2009”). ${ }^{14}$

6 Charles O. Jones, The Separated Presidency - Making it Work in Contemporary Politics, in: Anthony King (Hrsg.), The New American Political System, Washington D.C. 1990, S. 1 - 28, S. 3.

7 Sergio Fabbrini, The American System of Separated Government: An Historical-Institutional Interpretation, in: International Political Science Review, 20. Jg. (1999), H. 1, S. 95 - 116, S. 113.

8 Ebenda. Die Einschätzung Fabbrinis deckt sich mit der Einordnung in Shugart und Careys Klassifizierungsmuster „Regimes with Elected Presidents”; Matthew Soberg Shugart / John M. Carey, a.a.O. (Fn. 1), S. $18-27$.

9 Als Schlaglichter bezüglich der Stellung des Präsidenten gegenüber der Legislative zählen die folgenden Urteile des Supreme Courts: Youngstown Sheet \& Tube Co v. Sawyer (1952), United States v. Nixon (1974), INS v. Chadha (1983), Bowsher v. Synar (1986); vgl. Duane Lockard I Walter F. Murphy, Basic Cases in Constitutional Law, Washington D.C. 1987, S. 35 - 66.

10 William J. Clinton v. City of New York, 25. Juni 1998, http://laws.findlaw.com/us/000/97-1374. html (Abruf am 5. Dezember 2007).

11 Vgl. Majid Sattar, Formale und informale Politik. Wandlungen des Legislativ-Exekutiv-Verhältnisses am Beispiel der parlamentarischen Kontrollfunktion im amerikanischen Regierungssystem, Berlin 2001, S. 125.

12 Vgl. The New York Times, Transcript of President Bush's News Conference, 5. November 2004; The Washington Post, President Bush's State of the Union Address, 31. Januar 2006.

13 Vgl. Oxford Analytica, GOP Presidential Candidates Grapple with Tax-Cut Theory and Reality, in: The Hill vom 24. Oktober 2007.

14 Vgl. Walter Alarkon, White House Taking a Step Back on Line-Item Veto Power, in: The Hill vom 4. März 2009. 


\section{Das präsidentielle Line-Item Veto}

\subsection{Ausgangslage}

Jedes Kongressmitglied entscheidet für die gesamte Nation. ${ }^{15}$ Ebenso wird ein Abgeordneter oder ein Senator Einzel- und Sonderinteressen in den parlamentarischen Willensbildungsprozess mit einbringen, folgt man Winfried Steffani, mitunter ,ausdrücklich müssen" ${ }^{16}$. Dies gilt insbesondere für die Anliegen jener Wähler, deren Unterstützung für die Wiederwahl eines Abgeordneten wesentlich ist. ${ }^{17}$ Vor diesem Hintergrund ermutigt das institutionelle Arrangement Kongressmitglieder, Haushaltspolitik dafür einzusetzen, den Forderungen ihrer Wählerschaft - auch auf Kosten fiskalischer Verantwortung - zu entsprechen. ${ }^{18}$ Sie versuchen, staatliche Investitionen in ihre Wahlkreise zu lenken, so genanntes „pork“. Entsprechend werden die Verhandlungen im Kongress darüber als „pork barrel legislation “ bezeichnet. ${ }^{19}$ Gegen derartige Klientelpolitik war die Einführung des selektiven Vetos gerichtet, das dem Präsidenten das Recht gab, bestimmte Gesetzespassagen zu streichen, ohne gleich das ganze Gesetz blockieren zu müssen. So konnte der Präsident Gefälligkeitsausgaben verhindern, die nur einer eng umrissenen Empfängergruppe zugute kamen. ${ }^{20}$

Erfahrungen mit einem selektiven Veto sind auf der Ebene der amerikanischen Einzelstaaten weit verbreitet. 43 der 50 Gouverneure besitzen in der einen oder anderen Form das Recht, ihr Veto auch gegen einzelne Finanzposten aus Haushaltsgesetzen einzulegen (Gubernatorial Veto). ${ }^{21}$ Aus dieser Perspektive erscheint die Diskussion um das Line-Item Veto auf bundesstaatlicher Ebene keineswegs überraschend. Schon Präsident Ulysses S. Grant for-

15 Vgl. Winfried Steffani, Der Kongress, in: Wolfgang Jäger / Wolfgang Welz (Hrsg.), Regierungssystem der USA, München / Wien 1995, S. 110 - 135, S. $131 \mathrm{f}$.

16 Ebenda, S. 132. Die unterschiedlichen Arbeitsprofile der Repräsentanten haben Fiorina und Rhode als Hill Style (in Anlehnung an den Standort des Kongresses auf dem Capitol Hill) und Home Style geprägt; Morris P. Fiorina / David W. Rhode (Hrsg.), Homestyle and Washington Work, New York 1994.

17 Vgl. Richard F. Fenno, Home Style: House Members in Their Districts, New York 2003; John W. Kingdon, Congressmen's Voting Decisions, Ann Arbor 1989; R. Douglas Arnold, The Logic of Congressional Action, New Haven / London 1992; David R. Mayhew argumentiert, dass die grundsätzliche Motivation eines Abgeordneten die Wiederwahl sei und das Streben nach diesem Ziel bestimme die Weise, wie sie sich verhalten und Politik machen; David R. Mayhew, Congress: The Electoral Connection, New Haven / London 2004.

18 Vgl. Matthew J. Gabel / Gregory L. Hager, How to Succeed at Increasing Spending Without Really Trying: The Balanced Budget Amendment and the Item Veto, in: Public Choice, 102. Jg. (2000), H. 1, S. 19 - 23, S. 19.

19 Vgl. Richard S. Katz, Political Institutions in the United States, Oxford 2007, S. 159; Walter J. Oleszek, Congressional Procedures and the Policy Process, Washington D.C. 2007, S. 47.

20 Vgl. Isabel Sawhill / Ron Haskins, The Politics of Deficit Reduction, in: Alice Rivlin / Isabel Sawhill (Hrsg.), Restoring Fiscal Sanity: Meeting the Long-Run Challenge, Washington D.C. 2005, S. 119 - 138, S. 131.

21 Bei den sieben Bundesstaaten, in denen die Gouverneure nicht über das Recht eines Line-Item Vetos verfügen, handelt es sich um Indiana, Maryland, Nevada, New Hampshire, North Carolina, Rhode Island und Vermont. Nähere Informationen findet man auf der Website der National Conference of State Legislatures, http://www.ncsl.org/programs/fiscal/lbptabls/lbpc6t3.htm (Abruf am 20. März 2007). 
derte 1876 die Kompetenz, sich nicht mehr nur gegen komplette Bewilligungsgesetze aussprechen zu können, sondern auch einzelne Passagen aus Haushaltsgesetzen zu streichen. ${ }^{22}$ Erst Präsident Clinton erhielt am 1. Januar 1997 dieses Recht. ${ }^{23}$

John McCain hob vor der Einführung des Line-Item Vetos das Attribut der Haushaltskonsolidierung besonders hervor: "The line-item veto is not a means to encourage presidential abuse, but a means to end congressional abuse. It will give the President appropriate power to help control spending and reduce the deficit." ${ }^{24}$

\subsection{Entstehung des Gesetzes}

Anlässlich des Wahlkampfes zu den Kongresswahlen im November 1994, die in der Mitte der ersten Amtsperiode von Präsident Clinton stattfanden, veröffentlichte die Republikanische Partei den „Contract with America“. ${ }^{25}$ Mit dem unter Führung von Newt Gingrich initiierten Bundeswahlprogramm sollte es gelingen, den Kongress, der über sechs Jahrzehnte von den Demokraten geprägt worden $w^{26}{ }^{26}$, zurück zu gewinnen. Das Programm schilderte detailliert die Politikinhalte, die die Republikanischen Kandidaten für den Fall umzusetzen versprachen, dass sie die Mehrheit im Repräsentantenhaus stellen würden. ${ }^{27}$ Darunter befand sich der Fiscal Responsibility Act. Er sah einen Verfassungsanhang (Constitutional Amendment) vor, der die Pflicht zu einem ausgeglichenen Haushalt festschreiben sowie den Präsidenten mit dem Line-Item Veto ausstatten sollte. Letzteres war die einzige Komponente des Republikanischen Contract with America, die der Demokratische Präsident Clinton unterstützte.

Nach dem Wahlerfolg der Republikaner im November 1994 wurden einige der aufgezählten Gesetzentwürfe eingebracht ${ }^{28}$; darunter befand sich auch das selektive Veto für den Präsidenten im Haushaltsverfahren. ${ }^{29}$ Es wurden drei Varianten diskutiert: das Separate

22 Vgl. Virgina A. McMurtry, Item Veto and Expanded Impoundment Proposals, CRS Issue Brief for Congress, IB89148, Washington D.C. 2006.

23 Line Item Veto Act, Public Law 104-130, 9. April 1996, http://frwebgate.access.gpo.gov/cgi-bin/ getdoc.cgi?dbname=104_cong_public_laws\&docid=f:publ130.104.pdf (Abruf am 20. März 2007).

24 John McCain, Congressional Record - Senate, 27. März 1996, S. S2932.

25 Die meisten Politikinhalte gehen auf die Heritage Foundation zurück, http://www.house.gov/ house/Contract/CONTRACT.html (Abruf am 14. Dezember 2007). Einen Einblick in deutscher Sprache gibt Claus Leggewie, America first? Der Fall einer konservativen Revolution, Frankfurt am Main 1997.

26 Die Demokratische Kontrolle beider Häuser des Kongresses währte von 1933 bis 1995 und wurde nur im 80. (1947/48) und 83. Kongress (1953/54) sowie durch die Republikanische Mehrheit im Senat zwischen 1981 und 1987 durchbrochen.

27 Vgl. Patrick Horst, Der neue Republikanische US-Kongress: Polarisiert, zentralisiert und nachgiebig gegenüber dem Präsidenten, in: ZParl, 36. Jg. (2005), H. 3, S. 680 - 699, S. 686 f.; Donald $R$. Wolfensberger, The House That Newt Rebuilt: Or Did He?, in: APSA Legislative Studies Section Newsletter, Extension of Remarks, 28. Jg. (2005), H. 1, S. 3 - 7.

28 Vgl. J.F. Watts, The Republican Party. 1993-2001, in: Arthur M. Schlesinger, Jr. (Hrsg.), History of U.S. Political Parties. 1972-2001 The Politics of Consensus, Bd. V, Philadelphia 2002, S. 3853 - 4006, S. 3863.

29 Im Repräsentantenhaus brachten vier Republikanische Abgeordnete den Gesetzentwurf H.R.2 (,To give the President item veto authority over appropriation acts and targeted tax benefits in revenue acts”, kurz „Line-Item Veto Act“) ein, http://thomas.loc.gov/cgi-bin/bdquery/z?d104: 
Enrollment-, das Enhanced Rescission- und das Expedited Rescission-Verfahren. Der Senat favorisierte ersteres. Hierbei werden Haushaltsgesetze durch den Schriftführer des Parlaments in viele kleine Gesetze (Mini-Bills) zerteilt, bevor sie dem Präsident zur Unterschrift vorgelegt werden. Jeder einzelne Geldbetrag stellt damit ein separates Gesetz dar, das der Präsident mit seinem Veto belegen kann. ${ }^{30}$ Die Mitglieder des Repräsentantenhauses zogen hingegen das Enhanced Rescission-Verfahren vor. Hier schlägt der Präsident Streichungen vor, die nur dann keine Gültigkeit erlangen, wenn der Kongress ein missbilligendes Gesetz erlässt. ${ }^{31}$ Die Parlamentarier diskutierten auch das Expedited Rescission-Verfahren. Es garantiert dem Präsidenten, dass die von ihm vorgeschlagenen Haushaltskürzungen dem Kongress zur Abstimmung vorgelegt werden. Lehnt nur eine Kammer die Streichungen ab, treten sie nicht in Kraft. ${ }^{32}$

Die Republikaner wollten dem Demokratischen Präsidenten Clinton bei der Wahl des Verfahrens - gerade zu Beginn der Verhandlungen für den Bundeshaushalt 1996 - nicht zuviel Macht übertragen. Nach zähen Verhandlungen in den Ausschüssen, der Beilegung des inhaltlichen Streits über die drei Varianten innerhalb der Republikanischen Fraktion und der Verhinderung eines drohenden Filibusters durch Demokratische Senatoren ${ }^{33}$ brachte die Konsultierung des Vermittlungsausschusses im Frühjahr 1996 den Durchbruch $^{34}$ : Die Beteiligten einigten sich auf das Enhanced Rescission-Verfahren unter der Bedingung, dass das Gesetz zum 1. Januar 2005 wieder außer Kraft trete (Sunset Clause). Der Senat nahm den gemeinsamen Text für den lange umstrittenen Line-Item Veto Act am 27. März 1996 mit 69 zu 31 Stimmen an (16 der 28 Mitglieder des Senate Committee on Appropriations stimmten dagegen). ${ }^{35}$ Das Repräsentantenhaus folgte dieser Abstimmung am nächsten Tag mit 328 zu 91 Stimmen bei zwölf Enthaltungen. ${ }^{36}$

Präsident Clinton unterschrieb das Gesetz (P.L. 104-13037) am 9. April 1996. Zum 1. Januar 1997 - kurz vor Beginn seiner zweiten Amtszeit - trat es in Kraft. Zur Unterzeichnung äußerte sich Clinton wie folgt (Signing Statement): „This new authority brings us closer to the Founders' view of an effective executive role in the legislative process. The President will be able to prevent the Congress from enacting special interest provisions." 38

HR00002:@@@L\&umm2=m\& (Abruf am 19. Februar 2008). Im Senat initiierte der Republikanische Senator Robert J. Dole (Kansas) den Gesetzentwurf S.4 („A bill to grant the power to the President to reduce budget authority“, kurz „Line-Item Veto Act of $1995^{“}$ ), http://thomas.loc. gov/cgi-bin/bdquery/z?d104:SN00004:@@@Lsumm2=m\& (Abruf am 19. Februar 2008).

30 Vgl. Virgina A. McMurtry, House and Senate Votes on Proposals to Give the President Item Veto or Expanded Item Veto Authority, CRS Staff Memorandum, Washington D.C. 1995.

31 Vgl. Philip G. Joyce / Robert D. Reischauer, The Federal Line-Item Veto: What Is It and What Will It Do?, in: Public Administration Review, 57. Jg. (1997), H. 2, S. 95 - 104, S. 96.

32 Vgl. Virgina A. McMurtry, Presidential Rescission Authority: Efforts to Modify the 1974 Framework, CRS Report for Congress, RL30223, Washington D.C. 2004, S. 1.

33 Vgl. Andrew Taylor, In the Senate, A GOP Standoff, in: Congressional Quarterly Weekly Report vom 11. Februar 1995, S. 442.

34 Conference Report, H.Rept. 104-491, http://thomas.loc.gov/cgi-bin/cpquery/R?cp104:FLD010: @1(hr491) (Abruf am 19. Februar 2008).

35 Congressional Record - Senate, 27. März 1996, Rollcall Vote No. 56, S. S2995.

36 Congressional Record - House, 28. März 1996, Rollcall Vote No. 102, S. H3028.

37 Line Item Veto Act, a.a.O. (Fn. 23).

38 Bill Clinton, Statement by the President on Line Item Veto, 9. April 1996, http://clinton6.nara. gov/1995/03/1995-04-09-statement-by-president-on-line-item-veto.html (Abruf am 29. Februar 2008). 


\subsection{Neue Befugnis im Haushaltsverfahren}

Im Gewaltenteilungssystem der USA stellt der Kongress eine Legislative im strengen Sinne des Wortes dar (Artikel I, Abschnitt 1 US-Verfassung). ${ }^{39}$ Seine Gesetzgebungskompetenzen sind in der amerikanischen Verfassung in Artikel I, Abschnitt 8 festgelegt, der eine längere, achtzehn Punkte umfassende Aufzählung der Befugnisse enthält (Enumerated Powers). Dort wird dem Kongress auch das Recht eingeräumt, alle Gesetze zu erlassen, die er für nützlich und angemessen erachtet, um seinen aufgelisteten Gesetzgebungskompetenzen nachkommen zu können (Necessary and Proper Clause). ${ }^{40}$ Von ganz außerordentlicher Bedeutung ist jene in der Bundesverfassung ausdrücklich genannte Kongressbefugnis, die in Artikel I, Abschnitt 9, Absatz 7 festgelegt ist: „No money shall be drawn from the Treasury but in consequence of appropriations made by law [...]." Damit erhält der Kongress nicht nur die Kompetenz, generelle Bundesgesetze zu verabschieden, sondern auch genauestens zu bestimmen, wann, zu welchem Zweck und wie viele Gelder der Staatskasse entnommen werden dürfen (Power of the Purse). ${ }^{41}$

Folgt man der Verfassung, führt der Präsident die vom Kongress beschlossenen Gesetze aus (Artikel II, Abschnitt 1). ${ }^{42}$ Seine politische Führung äußert sich aber auch in seiner Einflussnahme auf das Gesetzgebungsverfahren. ${ }^{43}$ Formal bieten sich ihm drei Möglichkeiten: der Einfluss auf die Kongresssessionen, die Möglichkeit von Botschaften an die beiden Häuser und die Befugnis, Gesetzesvorlagen mit einem Veto zu belegen. ${ }^{44}$ Das Veto findet sich nicht im Abschnitt mit den Rechten des Präsidenten, sondern ist recht versteckt im Legislativartikel aufgeführt (Artikel I, Abschnitt 7, Absatz 2). James L. Sundquist macht jedoch die Tragweite des Präsidialvetos deutlich: „The most important of all the checks and balances is, of course, the presidential veto. “ ${ }^{55}$ Gesetze, denen der Präsident zustimmt, versieht er mit seiner Unterschrift; solche, die er missbilligt, kann er mit dem Veto belegen

39 Vgl. Christopher J. Bailey, The US Congress, New York 1989, S. 7 - 18; Winfried Steffani, Der Gesetzgebungsprozess, in: Wolfgang Jäger / Wolfgang Welz (Hrsg.), a.a.O. (Fn. 15), S. $184-197$, S. 184.

40 Vgl. Steffen Schmidt / Mack Shelley / Barbara A. Bardes, American Government and Politics Today, Belmont 2003, S. 214 f.

41 Vgl. Richard F. Fenno, The Power of the Purse. Appropriations Politics in Congress, Boston 1966; Winfried Steffani, a.a.O. (Fn. 15), S. 116.

42 Vgl. James P. Pfiffner / Roger H. Davidson, Understanding the Presidency, Longman 2006; James P. Pfiffner, The Modern Presidency, Belmont 2008.

43 Vgl. Jon R. Bond / Richard Fleisher, The President in the Legislative Arena, Chicago 1990; Michael Foley / John E. Owens, Congress and the Presidency. Institutional Politics in a Separated System, Manchester / New York 1996, S. 99; Louis Fisher, Constitutional Conflicts Between Congress and the President, Lawrence 1997, S. $119 \mathrm{ff}$.

44 Vgl. Louis Fisher, The Politics of Shared Power. Congress and the Executive, Washington D.C. 1998, S. 25; Karl Loewenstein, Verfassungsrecht und Verfassungspraxis der Vereinigten Staaten, Berlin u.a. 1959, S. 368 ff. Darüber hinaus können weitere Befugnisse des Präsidenten, wenn auch nicht in der Verfassung benannt, aus ihr abgeleitet werden (Inherent Powers); vgl. dazu die Debatte in der Zeitschrift Presidential Studies Quarterly, 37. Jg. (2007), H. 1: Louis Fisher, Invoking Inherent Powers: A Primer, S. 1 - 22; Neil Kinkopf, Inherent Presidential Power and Constitutional Structure, S. 37 - 48; Richard M. Pious, Inherent War and Executive Powers and Prerogative Politics, S. $66-84$.

45 James L. Sundquist zitiert nach Robert J. Spitzer, The Presidential Veto: Touchstone of the American Presidency, New York 1988, S. 1. 
und dabei dem Kongress seine Einwendungen bekannt geben. Daraus resultiert, dass sowohl die Unterschriftsgewährung als auch ihre Verweigerung den Präsidenten verfassungsrechtlich an der Gesetzgebungsfunktion beteiligen. Das Veto bedeutet dabei keinen absolut gültigen Einspruch, sondern hat nur aufschiebende Wirkung. Falls der Kongress mit Zweidritteln beschließt, das Veto außer Kraft zu setzen (Override), erlangt das Gesetz auch ohne die Zustimmung des Präsidenten Gültigkeit.

Die Verabschiedung des Bundeshaushalts weicht in den USA vom üblichen Gesetzgebungsprozess ab. ${ }^{46}$ Grundlage für die Aufstellung des Haushalts sind der Budget and Accounting Act von $1921^{47}$ und der Congressional Budget and Impoundment Control Act (CBICA) aus dem Jahr 1974. ${ }^{48}$

Geldausgaben der Bundesregierung müssen im Kongress einen zweistufigen Prozess durchlaufen, bevor sie im Prinzip denselben Weg nehmen wie andere Gesetzesinitiativen. Ein erstes Gesetz bestimmt zunächst Inhalt und Obergrenzen für Bundesbehörden und Ausgabenprogramme (Authorization), während bei der Verabschiedung der Bewilligungsgesetze (Appropriation) die Summe der Ausgaben festgelegt wird. ${ }^{49}$ Der CBICA gibt dem Präsidenten die Möglichkeit, die Ausgabe von Geldmitteln aufzuschieben (Deferral Authority) oder zu streichen (Rescission Authority). ${ }^{50}$ Wenn Senat und Repräsentantenhaus dem nicht innerhalb von 45 Tagen zustimmen, müssen die Gelder dem vorgesehenen Zweck zugeführt werden. Dem Kongress steht es frei, ob er über die Intervention des Präsidenten eine Abstimmung durchführt; in den meisten Fällen hat er sie bisher ignoriert. Der CBICA wurde als Antwort auf die wiederholte Weigerung Präsident Richard M. Nixons, bewilligte Mittel zu verwenden, eingeführt. ${ }^{51}$ Als Gegenreaktion wurde immer wieder - aber bis zur Amtszeit Clintons erfolglos - die Einführung eines selektiven Vetos vorgeschlagen, das dem Präsidenten ermöglichen sollte, bestimmte Ausgabeposten aus Gesetzen zu streichen. ${ }^{52}$ Der Line-Item Veto Act von 1996 ergänzte somit die Befugnisse des Präsidenten, in die Ausgabenpolitik einzugreifen.

Das Enhanced Rescission-Verfahren erlaubte dem Präsidenten „(1) any dollar amount of discretionary budget authority, (2) any item of new direct spending, and (3) certain limited tax benefits “53 aus Haushaltsgesetzen zu streichen (Cancellation). Die Annullierungen wa-

46 Vgl. Lance T. LeLoup, Budgetary Politics, Brunswick 1980, S. 152 - 182.

47 Der Budget and Accounting Act von 1921 richtete den Rechnungshof (General Accounting Office) ein. Die unabhängige Behörde wurde als investigativer Arm an den Kongress angegliedert. Im Jahr 2004 wurde der Name des Rechnungshofes in Government Accountability Office geändert.

48 Der Congressional Budget Act of 1974 legt das Verfahren zur Erstellung des Haushaltsplans fest. Das Gesetz wurde wiederholt geändert, so zum Beispiel durch den Balanced Budget and Emergency Deficit Control Act of 1985 und den Budget Enforcement Act of 1990. Die Vorgaben des ursprünglichen Gesetzes sind aber bis heute erhalten geblieben.

49 Vgl. Lance T. LeLoup, a.a.O. (Fn. 46), S. 184 -210; Winand Gellner / Martin Kleiber, Das Regierungssystem der USA, Baden-Baden 2007, S. 53.

50 Vgl. William G. Dauster, Budget Process Law Annotated, Washington D.C. 1993; Alan S. Frumin, Congressional Budget, Washington D.C. 1992, S. $502-642$.

51 Vgl. Robert Keith / Allen Schick, Introduction to the Federal Budget Process, CRS Report for Congress, 98-721 GOV, Washington D.C. 2004; Virgina A. McMurtry, a.a.O. (Fn. 32), S. 1.

52 Vgl. Virgina A. McMurtry, Item Veto and Expanded Impoundment Proposals: Legislative History and Current Status, CRS Report for Congress, RL33635, Washington D.C. 2006, S. 6 ff.

53 Vgl. Line-Item Veto Act, a.a.O. (Fn. 23). 
ren nur möglich, wenn sie die Staatsverschuldung senken, keine wesentliche Funktion der Regierung beinträchtigen und nicht mit dem nationalen Interesse kollidieren. ${ }^{54}$ Nach einer Streichung war der Präsident aufgefordert innerhalb von fünf Tagen (Sonntage ausgeschlossen) den Kongress zu informieren. Für jedes Gesetz musste eine spezielle Nachricht an die Haushaltsausschüsse und andere betroffene Ausschüsse des Repräsentantenhauses und des Senats gesendet werden. Sie führte die betreffenden Passagen, den Grund ihrer Streichung und Fristen auf. Diese Streichung trat in Kraft, wenn der Kongress nicht in beiden Häusern mit einfacher Mehrheit eine Resolution verabschiedete, die das präsidentielle Ansinnen ablehnte (Disapproval Resolution). In diesem Fall wurden alle Streichungen des Präsidenten null und nichtig.

Anstatt vom Präsidenten wie zuvor die Unterstützung des Kongresses innerhalb einer bestimmten Zeitspanne zu verlangen, kehrte der Line-Item Veto Act das bestehende Verfahren um. Die Anstrengung lag nun bei der Legislative, die Forderung des Präsidenten in einem beschleunigten Verfahren binnen 30 Tagen zu missbilligen. ${ }^{55}$ Gegen die Disapproval Resolution konnte der Präsident allerdings wiederum sein verfassungsmäßig gewährleistetes Veto einlegen, das schließlich mit einer Zweidrittelmehrheit in beiden Häusern überstimmt werden konnte (vgl. Abbildung 1).

Somit konnten letztlich bewilligte Gelder gegen den Willen der (einfachen) Mehrheit im Kongress vom Präsidenten gestrichen werden ${ }^{56}$ - eine deutliche Schwächung des legislativen Haushaltsrechts. Der Kongress hatte mit seinem Beschluss für die Ausweitung der präsidentiellen Befugnisse selbst das Gleichgewicht der Gewaltenteilung ins Wanken gebracht. ${ }^{57}$

\section{Die Anwendung des Line-Item Veto}

Als am 1. Januar 1997 der Line-Item Veto Act in Kraft trat, erhielt Präsident Clinton ein neues Werkzeug im Haushaltsprozess. Weniger als achtzehn Monate später am 25. Juni 1998, erklärte der Oberste Gerichtshof der USA die neue institutionelle Kompetenz des Präsidenten für verfassungswidrig. Nach dieser Entscheidung blieb die Line-Item VetoKompetenz des Präsidenten weitestgehend unerforscht: Wie oft und mit welchen Konsequenzen nutzte er sie? Aus programmatischen oder parteipolitischen Erwägungen oder aus Gründen der - bei Einführung intendierten - Haushaltskonsolidierung?

\subsection{Häufigkeit der Anwendung}

Zwischen dem Inkrafttreten und dem Urteil des Obersten Gerichtshofs strich der Präsident in elf Gesetzesvorlagen 82 Passagen (vgl. Tabelle 1). Der Kongress ging unterschiedlich mit den präsidentiellen Streichungen um. Neben der einfachen Bestätigung machte er durchaus

54 Ebenda.

55 Vgl. Louis Fisher / Virgina A. McMurtry, The Line Item Veto Act: Procedural Issues, CRS Report for Congress, 96-973 GOV, Washington D.C. 1996, S. 1.

56 Vgl. Majid Sattar, a.a.O. (Fn. 11), S. 126.

57 Vgl. Philip G. Joyce / Robert D. Reischauer, a.a.O. (Fn. 31), S. 95 ff. 


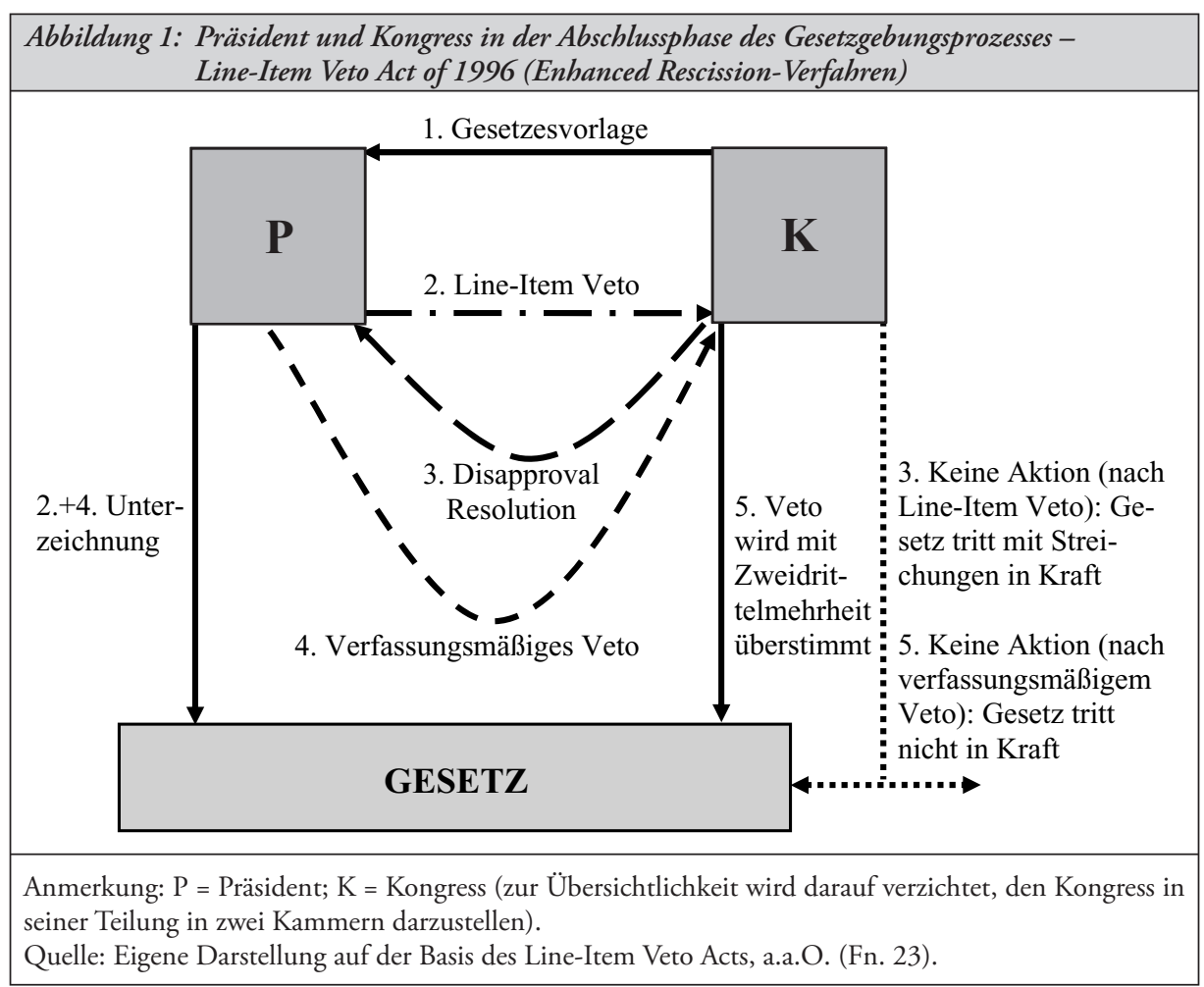

Gebrauch davon, die Annullierungen der Exekutive mittels Resolution abzulehnen, wohl wissend, damit Gefahr zu laufen, vom althergebrachten Veto des Präsidenten überstimmt zu werden. In zwei Fällen, den Bewilligungsgesetzen für das Innen- und das Agrarressort (Department of Interior and Related Agencies Appropriations Act of 1998 sowie Agriculture, Rural Development, Food and Drug Administration, and Related Agencies Appropriations Act of 1998), zog der Kongress die Annullierungen in Betracht und modifizierte die Gesetzesvorlagen in Abstimmung mit der Exekutive in den zuständigen Ausschüssen, bevor sie dem Präsidenten erneut zur Unterschrift vorgelegt wurden. ${ }^{58}$

Zu einem ersten Konflikt zwischen Kongress und Präsident kam es im Herbst 1997 über den Military Construction Appropriations Act of $1998 .{ }^{59}$ Die Parlamentarier missbilligten vor allem, dass Clinton 38 militärische Bauprojekte im Wert von 287 Millionen Dollar aus dem Haushalt für das nächste Jahr herausgestrichen hatte. Der Kongress revidierte kurzerhand die Streichungen durch eine Disapproval Resolution. Dagegen legte Clinton sein präsidentielles Veto ein, das unmittelbar mit einer Zweidrittelmehrheit überstimmt wurde. ${ }^{60}$

58 Vgl. Majid Sattar, a.a.O. (Fn. 11), S. 126 f.

59 Vgl. M. V. Hood, III / Irwin L. Morris / Grant W. Neeley, Penny Pinching or Politics? The Line Item Veto and Military Construction Appropriations, in: Political Research Quarterly, 52. Jg. (1999), H. 4, S. $753-766$.

60 Vgl. ebenda. 


\begin{tabular}{|c|c|c|c|c|c|c|c|c|c|c|c|c|c|}
\hline$\frac{3}{\pi}$ & 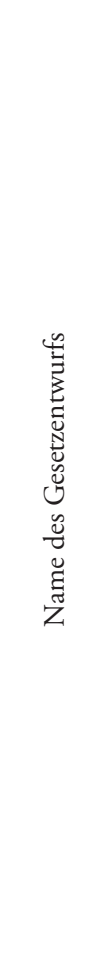 & 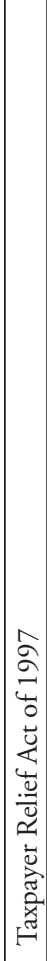 & 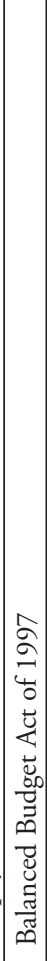 & 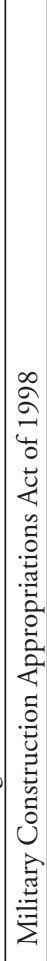 & 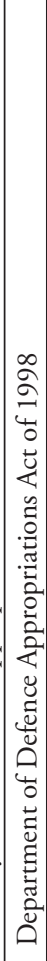 & 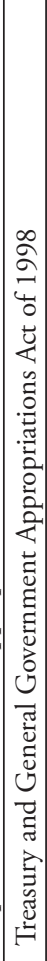 & 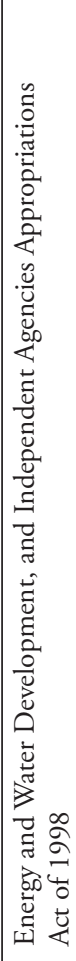 & 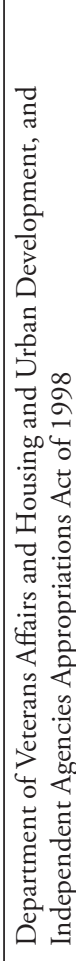 & 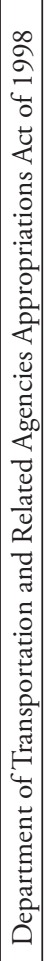 & 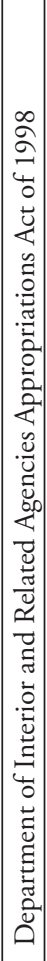 & 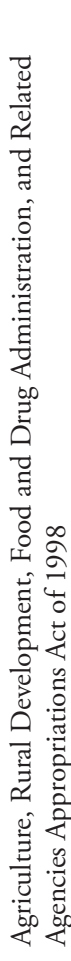 & 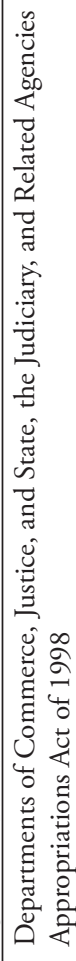 & 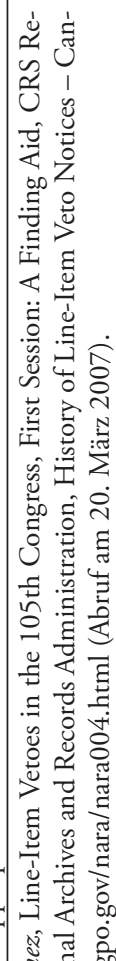 \\
\hline 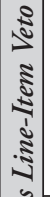 & 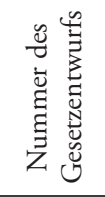 & 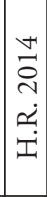 & 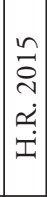 & 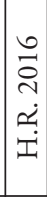 & 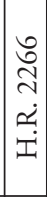 & 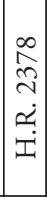 & 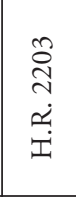 & $\begin{array}{l}\infty \\
\stackrel{n}{n} \\
\dot{\sim} \\
\dot{I} \\
\dot{I}\end{array}$ & 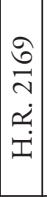 & 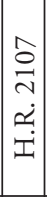 & 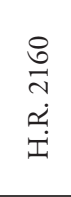 & 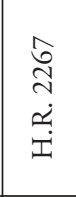 & 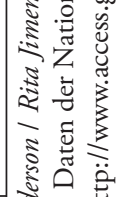 \\
\hline 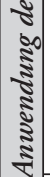 & 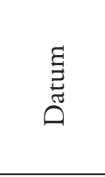 & 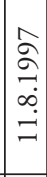 & 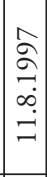 & 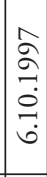 & 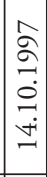 & $\mid \begin{array}{l}2 \\
2 \\
\vdots \\
0 \\
-1 \\
0 \\
-1\end{array}$ & $\begin{array}{l}2 \\
\stackrel{2}{0} \\
\stackrel{0}{1}\end{array}$ & 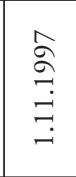 & 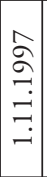 & 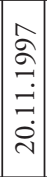 & 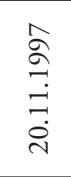 & ڤ્ & 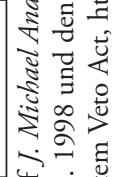 \\
\hline 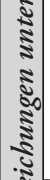 & 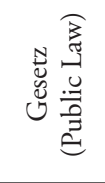 & 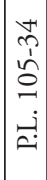 & 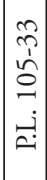 & 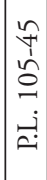 & 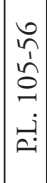 & 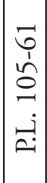 & 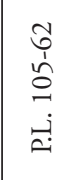 & \begin{tabular}{l}
\multirow{n}{0}{} \\
$\hat{1}$ \\
0 \\
-1 \\
$\dot{1}$
\end{tabular} & 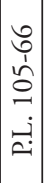 & 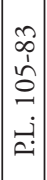 & 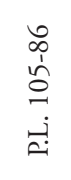 & 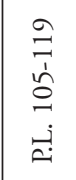 & 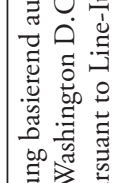 \\
\hline 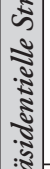 & 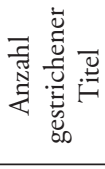 & $\sim$ & - & $\stackrel{\infty}{m}$ & \pm & -1 & $\infty$ & $\Lambda$ & $m$ & $\sim$ & $n$ & - & 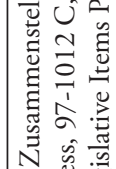 \\
\hline 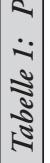 & 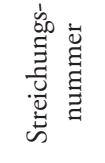 & $\stackrel{T}{-1}$ & $n$ & $\$$ & \begin{tabular}{l}
$\tilde{\sim}$ \\
\multirow{\sim}{*}{}
\end{tabular} & $\stackrel{゚}{\curvearrowleft}$ & $\begin{array}{l}0^{1} \\
\hat{n}\end{array}$ & ถ่' & $\begin{array}{l}\stackrel{+}{\hat{1}} \\
\stackrel{N}{N}\end{array}$ & $\begin{array}{l}\hat{n} \\
n \\
n\end{array}$ & $\begin{array}{l}\text { o } \\
\stackrel{1}{N}\end{array}$ & $\infty$ & 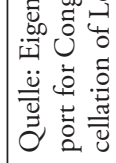 \\
\hline
\end{tabular}


Generell stellt sich die Frage, ob der Präsident das selektive Veto öfter als sein konstitutionelles eingesetzt hat. Bei welchem war es für den Kongress schwieriger, es zu überstimmen? Wurden weniger oder mehr Gesetze (im zweiten Anlauf) verabschiedet (Override)? Zur Beantwortung wird der 105. Kongress (1997 bis 1999) - in dem der Präsident über das selektive Line-Item Veto verfügte - mit dem 104. (1995 bis 1997) und 106. Kongress (1999 bis 2001) - in denen der Präsident nur das herkömmliche konstitutionelle Veto be$\mathrm{saß}$ - verglichen. ${ }^{61}$

Die Erwartung der Federalists, das Veto werde vom Präsidenten nur selten und nur in absoluten Notfällen angewendet, bewahrheitete sich nur im ersten halben Jahrhundert nach der Gründung der Republik. Die ersten Präsidenten machten von diesem Recht in der Regel nur dann Gebrauch, wenn ihnen ein Gesetz verfassungswidrig oder technisch anfechtbar erschien. Erst der Demokrat Andrew Jackson (1829 bis 1837) benutzte es, um unliebsame Gesetze des Republikanischen Kongresses zu verhindern. ${ }^{62}$ Vergleicht man die drei Legislaturperioden miteinander, so ist zu erkennen, dass das konstitutionelle Veto vom Präsidenten sparsam eingesetzt wurde (vgl. Tabelle 2). Seit Mitte der 1950er Jahre sind im Schnitt zehn Vetos pro Jahr eingelegt worden. Von 1995 bis 2001 waren es sogar noch weniger. Der sparsame Einsatz erklärt sich zum einen aus der indirekten Beteiligung des Präsidenten am Gesetzgebungsprozess und zum anderen dadurch, dass ein Veto auch der Öffentlichkeit vermittelt werden muss. ${ }^{63}$ Die Betrachtung der Legislaturperioden zeigt jedoch auch, dass der Präsident durch das selektive Veto eine neue Machtbefugnis erhalten hat, von der er im gleichen Umfang wie vom konstitutionellen Veto Gebrauch gemacht hat.

\begin{tabular}{|c|c|c|c|c|}
\hline \multicolumn{5}{|c|}{ Tabelle 2: Anzahl der Vetos im Zeitraum von 1995 bis 2001} \\
\hline Legislaturperiode & $\begin{array}{c}\text { Anzahl der } \\
\text { konstitutionellen } \\
\text { Vetos }\end{array}$ & $\begin{array}{c}\text { Anzahl der } \\
\text { überstimmten } \\
\text { Vetos }\end{array}$ & $\begin{array}{l}\text { Anzahl der } \\
\text { Line-Item } \\
\text { Vetos }\end{array}$ & $\begin{array}{c}\text { Anzahl der } \\
\text { überstimmten } \\
\text { Line-Item Vetos }\end{array}$ \\
\hline 104. Kongress (1995-1997) & 17 & 1 & - & - \\
\hline 105. Kongress (1997-1999) & 8 & $1^{*}$ & 11 & $1^{*}$ \\
\hline 106. Kongress (1999-2001) & 9 & - & - & - \\
\hline \multicolumn{5}{|c|}{$\begin{array}{l}\text { * Bei dem überstimmten Veto (Override) im 105. Kongress handelt es sich um ein und dasselbe Veto } \\
\text { gegen die Streichungen im Military Construction Appropriations Act of } 1998 . \\
\text { Quelle: Eigene Zusammenstellung basierend auf Gary L. Galemore, President Clinton's Vetoes, CRS Re- } \\
\text { port for Congress, } 98-147 \text { GOV, Washington D.C. 2000; ders., Congressional Overrides of Presidential } \\
\text { Vetoes, CRS Report for Congress, 98-157 GOV, Washington D.C. } 2001 .\end{array}$} \\
\hline
\end{tabular}

Das Vetorecht des Präsidenten ist im Miteinander von Präsident und Kongress kein unwichtiger Faktor. Da ein Veto kaum überstimmt werden kann, fördert es die Kooperations-

61 Die Gegenüberstellung dieser Legislaturperioden drängt sich auf, nicht nur aus Gründen zeitlicher Nähe, sondern auch, weil sie gleiche Voraussetzungen bieten. Alle drei sind durch eine geteilte Regierungsmacht (Divided Government) - das Auseinanderfallen der Parteizugehörigkeit des amerikanischen Präsidenten und der Parteizugehörigkeit der Mehrheit des Kongresses - gekennzeichnet: dem demokratischen Präsidenten Clinton stand im Kongress in beiden Kammern eine Republikanische Mehrheit gegenüber.

62 Vgl. Karl Loewenstein, a.a.O. (Fn. 44), S. 375.

63 Vgl. Birgit Oldopp, Das politische System der USA, Wiesbaden 2005, S. 69 f. 
bereitschaft auf beiden Seiten. ${ }^{64}$ Vermittelt ein Präsident glaubhaft, dass eine Entscheidung mit ihm nicht machbar sei, wird der Kongress dies in aller Regel berücksichtigen. Wenn der Präsident sein Veto einlegt, wird de facto das Inkrafttreten eines Gesetzes verhindert, denn die hohe Hürde einer Zweidrittelmehrheit kann nur in wenigen Fällen genommen werden. Dies setzt eine Geschlossenheit voraus, die im Kongress nur selten zu erzielen ist. ${ }^{65}$ Von 1.484 eingelegten Vetos wurden 107 überstimmt (Stand 2002) ${ }^{66}$, und zwar lediglich 50 zwischen 1789 und 1933, aber im Zeitraum von 1933 bis zum Ende der Präsidentschaft Clintons immerhin in 57 Fällen. ${ }^{67}$ Von den elf Line-Item Vetos wurde nur ein einziges überstimmt; wie beim konstitutionellen Veto blieb es somit schwierig, die präsidentiellen Positionen im Kongress zu revidieren.

Das Veto des Präsidenten ist zu einem wesentlichen Machtinstrument des Präsidenten gegen den Kongress geworden. Angesichts der Seltenheit seiner Ausschaltung durch den Kongress, kann man wohl sagen, dass es sich, wenn von einer Botschaft begleitet, von einem nur aufschiebenden in einen endgültigen Einspruch verwandelt hat. Allein die Androhung des Vetos kann zuweilen für die Gewinnung von Verhandlungsbereitschaft des Kongresses eingesetzt werden. ${ }^{68}$

\subsection{Anwendung aus parteipolitischen Motiven}

Um festzustellen, ob der Demokratische Präsident Clinton seine neu erworbene Befugnis als ein parteipolitisches Instrumentarium eingesetzt hat, soll geprüft werden, welche Mitglieder des Kongresses von den präsidentiellen Annullierungen betroffen waren.

Betrachtet man nur die dreizehn Abgeordneten und Senatoren, denen mindestens zwei ihrer Projekte in Haushaltsgesetzen gestrichen wurden, dann fällt auf, dass sich unter ihnen neun Republikaner, aber nur vier Demokraten befanden (vgl. Tabelle 3).

Wird nicht nur die Anzahl der gestrichenen Projekte berücksichtigt, sondern auch, welche Abgeordneten die höchsten Einbußen für ihren Wahlkreis hinnehmen mussten, dann tritt unter den fünf Abgeordneten mit den größten Verlusten ein ausgeglicheneres Bild zutage (vgl. Tabelle 4).

Ein Blick in die gesamten Zahlen der von den Annullierungen betroffenen Mitglieder des Kongresses macht deutlich, dass es den Demokraten des Repräsentantenhauses besser als ihren Republikanischen Kollegen ergangen ist. Diese verzeichneten mit 316,2 Millionen Dollar doppelt so hohe Streichungen für ihre Wahlkreise wie die Demokratischen Abgeord-

64 Vgl. Charles M. Cameron, Veto Bargaining - Presidents and the Politics of Negative Power, Cambridge 2000; ders. I Nolan McCarty, Models of Vetos and Veto Bargaining, in: Annual Review of Political Science, 7. Jg. (2004), H. 1, S. $409-435$.

65 Vgl. Birgit Oldopp, a.a.O. (Fn. 63), S. 69.

66 Harold W. Stanley / Richard Niemi, Vital Statistics on American Politics 2003-2004, Washington D.C. 2003, S. 260.

67 Vgl. Mitchel A. Sollenberger, The Presidential Veto and Congressional Procedure, CRS Report for Congress, RS21750, Washington D.C. 2004, S. 3 f.; Gary L. Galemore, Congressional Overrides of Presidential Vetoes, CRS Report for Congress, 98-157 GOV, Washington D.C. 2001, S. 3.

68 Vgl. Ludger Helms, Präsident und Kongress in der legislativen Arena. Wandlungstendenzen amerikanischer Gewaltenteilung am Ende des 20. Jahrhunderts, in: ZParl, 30. Jg. (1999), H. 4, S. $841-864$, S. 848. 


\begin{tabular}{|c|c|c|c|}
\hline \multicolumn{4}{|c|}{$\begin{array}{l}\text { Tabelle 3: Präsidentielle Streichungen unter Anwendung des Line-Item Veto Acts nach Mitgliedern } \\
\text { des Kongresses und Anzabl der gestrichenen Projekte }\end{array}$} \\
\hline Mitglieder des Kongresses & $\begin{array}{c}\text { Partei- } \\
\text { zugehörigkeit }\end{array}$ & Staat & $\begin{array}{c}\text { Anzahl der } \\
\text { gestrichenen Projekte }\end{array}$ \\
\hline Rick Hill & $\mathrm{R}$ & Montana & 5 \\
\hline Michael Crapo & $\mathrm{R}$ & Idaho & 3 \\
\hline Jerry Lewis & $\mathrm{R}$ & Kalifornien & 3 \\
\hline John Murtha & $\mathrm{D}$ & Pennsylvania & 3 \\
\hline Steven Schiff & $\mathrm{R}$ & New Mexico & 3 \\
\hline Don Young & $\mathrm{R}$ & Alaska & 3 \\
\hline Robert Aderholt & $\mathrm{R}$ & Alabama & 2 \\
\hline Marcy Kaptur & $\mathrm{D}$ & Ohio & 2 \\
\hline Jim Kolbe & $\mathrm{R}$ & Arizona & 2 \\
\hline Mike Parker & $\mathrm{R}$ & Mississippi & 2 \\
\hline Owen Pickett & $\mathrm{D}$ & Virginia & 2 \\
\hline Bennie Thompson & $\mathrm{D}$ & Mississippi & 2 \\
\hline John Thune & $\mathrm{R}$ & South Dakota & 2 \\
\hline \multicolumn{4}{|c|}{$\begin{array}{l}\text { Anmerkungen: } \mathrm{R}=\text { Republikanische Partei; } \mathrm{D}=\text { Demokratische Partei. } \\
\text { Quelle: Eigene Zusammenstellung basierend auf Rick Rothacker, Few Satisfied with Inaugural Use of the } \\
\text { Line-Item Veto, in: The Washington Post vom 2. Dezember 1997; The Washington Post, Members Hit } \\
\text { with the Most Line-Item Vetoes, 2. Dezember 1997. }\end{array}$} \\
\hline
\end{tabular}

\begin{tabular}{|c|c|c|c|}
\hline $\begin{aligned} \text { Tabelle 4: } \begin{array}{r}\text { Präsidentielle St } \\
\text { des Kongresses u }\end{array} \\
\end{aligned}$ & $\begin{array}{l}\text { ngen unter } A \\
\text { rag der gestri }\end{array}$ & $\begin{array}{l}\text { dung des Lin } \\
\text { en Projekte }\end{array}$ & Veto Acts nach Mitgliedern \\
\hline Mitglieder des Kongresses & $\begin{array}{c}\text { Partei- } \\
\text { zugehörigkeit }\end{array}$ & Staat & \begin{tabular}{|l|} 
Betrag gestrichener Projekte \\
(in Millionen US-Dollar)
\end{tabular} \\
\hline Steven Schiff & $\mathrm{R}$ & New Mexico & 54,0 \\
\hline Brad Sherman & $\mathrm{D}$ & Kalifornien & 37,5 \\
\hline William Thomas & $\mathrm{R}$ & Kalifornien & 30,0 \\
\hline Owen Pickett & $\mathrm{D}$ & Virginia & 23,9 \\
\hline John Murtha & $\mathrm{D}$ & Pennsylvania & 23,0 \\
\hline $\begin{array}{l}\text { Anmerkungen: } \mathrm{R}=\text { Republik } \\
\text { Quelle: Eigene Zusammenste } \\
\text { Line-Item Veto, in: The Wash } \\
\text { Losses by Member and Distri }\end{array}$ & $\begin{array}{l}\text { e Partei; } \mathrm{D}= \\
\text { basierend auf } \\
\text { Post vom } 2 . \\
\text { Dezember } 19\end{array}$ & $\begin{array}{l}\text { kratische Parte } \\
\text { Rothacker, Few } \\
\text { nber 1997; Th }\end{array}$ & $\begin{array}{l}\text { ied with Inaugural Use of the } \\
\text { hington Post, Greatest Dollar }\end{array}$ \\
\hline
\end{tabular}

neten mit 154,5 Millionen Dollar. ${ }^{69}$ Im Senat zeichnete sich keine Tendenz für die eine oder andere Seite ab. Daran, dass die Annullierungen auf zuvor getroffene informale Aushandlungen zurückzuführen sind, lässt der frühere Direktor der Haushaltsbehörde (Congressional Budget Office, CBO) Robert Reischauer keinen Zweifel: „Nothing in this city can be treated in isolation. [...] Every action affects other issues in play. "70

Es bleibt festzuhalten: Es gibt einen Zusammenhang zwischen den Streichungen des Demokratischen Präsidenten und der Parteizugehörigkeit der von den Streichungen betrof-

69 Vgl. Rick Rothacker, Few Satisfied with Inaugural Use of the Line-Item Veto, in: The Washington Post vom 2. Dezember 1997.

70 Ebenda. 
fenen Abgeordneten. Republikanische Mitglieder des Kongresses waren in größerem Umfang betroffen als ihre Demokratischen Kollegen. Dieser Umstand wird jedoch auch davon getragen, dass sie im 105. Kongress in einer größeren Zahl im Kongress vertreten waren. Allein, ob Clinton die Streichungen bewusst auf Grundlage parteipolitischer Präferenzen getroffen hat, ist nicht zu belegen.

\subsection{Anwendung aus haushaltspolitischen Motiven}

Vor der Einführung des Line-Item Vetos auf bundesstaatlicher Ebene war es schwierig, die potentiellen Einsparungen durch dieses Instrument zu schätzen. ${ }^{71}$ Die Übertragbarkeit von Erkenntnissen aus den Einzelstaaten war umstritten. ${ }^{72}$ Messbare Effekte des Line-Item Vetos auf ein höheres Maß an finanzpolitischer Verantwortlichkeit auf Landesebene sind nur in geringem Umfang abzulesen. ${ }^{73}$

Von Experten wurde dem Line-Item Veto vor seiner Einführung nur begrenzt die Eigenschaft zugeschrieben, signifikant zur Haushaltskonsolidierung beitragen zu können. Während einer Anhörung vor dem Kongress im Jahr 1995 schilderte Alice Rivlin, Direktorin der Exekutivbehörde für Haushaltserstellung und -kontrolle (Office of Management and Budget, OMB), ihre Erwartungen zum selektiven Veto des Präsidenten: „I would think that it would be a relatively small proportion of discretionary spending that would be a candidate for a Line-Item Veto. [...] I don't believe that this would be a large dollar figure. It is likely to be a relatively small percentage of total spending." "44 Sowohl Robert Reischauer (CBO) als auch Harry Havens, stellvertretender Vorsitzender des Bundesrechnungshofes (General Accounting Office, GAO), schlossen sich dieser Meinung an und verwiesen dazu noch auf die Gefahren: „The greatest impact [...] would not be in savings but rather in the balance of power between the legislative and executive branches. Various item-veto or rescission proposals would represent a major shift of power from Congress to the President in an area that was reserved to Congress by the Constitution and which has historically been one of clear legislative primacy."75

Tabelle 5 zeigt das Ausmaß der Klientelpolitik der Abgeordneten in ihrer Rückkopplung an die Wählerschaft. Trotz selektiven Vetos stiegen die Ausgaben, die an bestimmte Projek-

71 Vgl. Virgina A. McMurtry, Item Veto: Budgetary Savings, CRS Report for Congress, RS22155, Washington D.C. 2005, S. 4.

72 Vgl. Louis Fisher / Neal Devins, How Successfully Can the States' Item Veto Be Transferred to the President?, in: The Georgetown Law Journal, 75. Jg. (1986), H. 1, S. 159 - 197; James J. Gosling, Wisconsin Item-Veto Lessons, in: Public Administration Review, 46. Jg. (1986), H. 4, S. 292 300, S. 292.

73 Vgl. Glenn Abney / Thomas P. Lauth, The Line-Item Veto in the States: An Instrument for Fiscal Restraint or an Instrument for Partisanship?, in: Public Administration Review, 45. Jg. (1985), H. 3, S. 372 - 377; David C. Nice, The Item Veto and Expenditure Restraint, in: The Journal of Politics, 50. Jg. (1988), H. 4, S. 487 - 499; Thomas P. Lauth, The Line-Item Veto in Government Budgeting, in: Public Budgeting and Finance, 16. Jg. (1996), H. 2, S. 97 - 111; Glenn Abney I Thomas P. Lauth, The Item Veto and Fiscal Responsibility, in: The Journal of Politics, 59. Jg. (1997), H. 3, S. $882-892$.

74 Vgl. Virgina A. McMurtry, a.a.O. (Fn. 71), S. 5 f.

75 Ebenda; vgl. auch CBO Memorandum, The Line Item Veto Act After One Year, Washington D.C. 1998. 
te der Parlamentarier (so genannte Earmarks) gekoppelt waren, weiter an. Der von den Streichungen durch das selektive Veto betroffene Demokratische Abgeordnete John Murtha (Pennsylvania), erwiderte auf Bestrebungen, die Bevorzugung des Wahlkreises des jeweiligen Abgeordneten (Pork-Barrel Legislation) einzuschränken, nur: „They [the White House] don't appreciate the importance of these projects to members."76

\begin{tabular}{|c|c|c|c|c|}
\hline $\begin{aligned} \text { Tabelle 5: } & \text { Earmarks in Ber } \\
& \text { US-Dollar) }\end{aligned}$ & setzer & tsjahr & 2000 & nen \\
\hline $\begin{array}{c}\text { Bewilligungsgesetze } \\
\text { (Appropriations) }\end{array}$ & 1994 & 1996 & 1998 & 2000 \\
\hline Agriculture & 313 & 211 & 284 & 359 \\
\hline Commerce-Justice-State & 253 & 171 & 275 & 361 \\
\hline Defense & 587 & 270 & 644 & 997 \\
\hline District of Columbia & - & - & 3 & 16 \\
\hline Energy and Water & 1.574 & 1.421 & 1.877 & 1.707 \\
\hline Foreign Operations & 38 & 53 & 81 & 69 \\
\hline Interior & 314 & 137 & 320 & 479 \\
\hline $\begin{array}{l}\text { Labor-Health and Human } \\
\text { Services-Education }\end{array}$ & 5 & 7 & 25 & 491 \\
\hline Legislative Branch & 1 & - & 1 & - \\
\hline Military Construction & 895 & 556 & 461 & 518 \\
\hline Transportation & 140 & 167 & 147 & 641 \\
\hline Treasury & 53 & 33 & 11 & 19 \\
\hline $\begin{array}{l}\text { Veteran Affairs-Housing and } \\
\text { Urban Development }\end{array}$ & 30 & 48 & 140 & 469 \\
\hline Gesamt & 4.202 & 3.084 & 4.269 & 6.126 \\
\hline $\begin{array}{l}\text { Anmerkung: Der Congression } \\
\text { cher Definitionen die Angabe } \\
\text { sistent sind. Auch spricht er s } \\
\text { Haushaltsjahrgesamtrechnung } \\
\text { Quelle: Allen Schick, The Fede } \\
\text { Earmarks in Appropriations A }\end{array}$ & $\begin{array}{l}\text { Service } \\
\text { rchgäng } \\
\text { die Auf } \\
\text { Politic }\end{array}$ & $\begin{array}{l}\text { darauf, } \\
\text { Bewill } \\
\text { ang der } \\
\text { Process, }\end{array}$ & $\begin{array}{l}\text { h Verw } \\
\text { tze unc } \\
\text { der U } \\
\text { on D.C }\end{array}$ & $\begin{array}{l}\text { nterschiedli- } \\
\text { tsjahre kon- } \\
\text { hüsse in der } \\
\text { 246; CRS, }\end{array}$ \\
\hline
\end{tabular}

Ein Anzeichen dafür, in welchem Maße Haushaltskonsolidierung durch ein Line-Item Veto gefördert werden kann, ergibt sich aus Tabelle 6. Sie veranschaulicht den Einsatz des selektiven Vetos in Bezug auf die Bewilligungsgesetze im Haushaltsjahr 1998. Präsident Clinton strich 77 Passagen aus Haushaltsgesetzen; die Streichungen stellten einen Gegenwert von 491 Millionen Dollar dar. Auch wenn einige Annullierungen nur kleine Millionenbeträge ausmachten, ist es immerhin von symbolischem Wert, dass mehr als die Hälfte der Bewilligungsgesetze Kürzungen enthielten. Wie bereits angesprochen, waren dabei die Streichungen im Military Construction Appropriations Act of 1998 die größte Einflussnahme Clintons in den Haushaltsprozess: Die Annullierungen machten drei Prozent des Gesetzes aus. Insgesamt belief sich der Anteil der annullierten Geldbeträge aber lediglich auf 0,06 Prozent des Haushaltsaufkommens und ist damit verschwindend gering. Die vorab getroffenen Einschätzungen der Experten wurden also bestätigt.

76 John Murtha, zitiert nach Rick Rothacker, a.a.O. (Fn. 69), S. 1. 


\begin{tabular}{|l|c|c|c|c|}
\hline $\begin{array}{c}\text { Tabelle 6: Anwendung des Line-Item Vetos in Bewilligungsgesetzen, Haushaltsjahr 1998 (in } \\
\text { Millionen US-Dollar) }\end{array}$ \\
$\begin{array}{c}\text { Bewilligungsgesetze } \\
\text { (Appropriations) }\end{array}$ & $\begin{array}{c}\text { Geldzuwei- } \\
\text { sungen }\end{array}$ & $\begin{array}{c}\text { Anzahl der } \\
\text { Streichungen } \\
\text { durch das } \\
\text { Line-Item Veto }\end{array}$ & $\begin{array}{c}\text { Annullierte } \\
\text { Geldbeträge } \\
\text { durch das } \\
\text { Line-Item Veto }\end{array}$ & $\begin{array}{c}\text { Anteil der } \\
\text { annullierten } \\
\text { Geldbeträge } \\
\text { (in Prozent) }\end{array}$ \\
\hline Agriculture & 49.749 & 5 & 2 & 0,0039 \\
\hline Commerce-Justice-State & 31.816 & 1 & 5 & 0,0157 \\
\hline Defense & 245.708 & 13 & 144 & 0,0581 \\
\hline District of Columbia & 4.972 & - & - & - \\
\hline Energy and Water & 21.152 & 8 & 19 & 0,0912 \\
\hline Foreign Operations & 13.190 & - & - & - \\
\hline Interior & 13.789 & 2 & 6 & 0,0450 \\
\hline $\begin{array}{l}\text { Labor-Health and Human } \\
\text { Services-Education }\end{array}$ & 268.013 & - & - & - \\
\hline Legislative Branch & 2.248 & - & - & - \\
\hline Military Construction & 9.183 & 38 & 287 & 3,1253 \\
\hline Transportation & 13.062 & 3 & 6 & 0,0477 \\
\hline Treasury & 25.587 & 1 & 8 & 0,0313 \\
\hline $\begin{array}{l}\text { Veteran Affairs-Housing and } \\
\text { Urban Development }\end{array}$ & 90.735 & 7 & 14 & 0,0154 \\
\hline Gesamt & 759.211 & 77 & 491 & 0,0621 \\
\hline $\begin{array}{l}\text { Quelle: Lance T. LeLoup / Carolyn N. Long / James N. Giordano, President Clinton' Fiscal 1998 Budget: } \\
\text { Political and Constitutional Paths to Balance, in: Public Budgeting and Finance, 18. Jg. (1998), H. } 1, \\
\text { S. 3-32, S. 25. }\end{array}$ & & & & \\
\hline
\end{tabular}

\subsection{Anwendung aus programmatischen Motiven}

Mithilfe des Line-Item Vetos kann der Präsident, Projekte mit vermeintlich niedriger nationaler Dringlichkeit oder allein lokaler Orientierung, die häufig ohne Anhörung oder Debatte in den Ausschüssen von Kongressmitgliedern eingefügt werden, verhindern oder wenigstens reduzieren. Diese gelangen durch die Klientelpolitik der Repräsentantenhausmitglieder als „Vertreter von Wahlkreisinteressen“ und Senatoren als „Vertreter von Einzelstaatsinteressen“ in das Haushaltsverfahren. ${ }^{77}$ Die Betrachtung des Präsidenten als „representative of the general interest" ${ }^{\prime 8}$, der sein Veto allein zum Wohle des Volkes einsetzt, einerseits und die Wahrnehmung von Klientelpolitik allein durch die Parlamentarier andererseits ist allerdings ungenügend.

Der Präsident verwendet das Line-Item Veto nicht nur dazu Streichungen in Haushaltsgesetzen vorzunehmen, sondern wird versuchen, in Verhandlungen mit Kongressmitglie-

77 Winfried Steffani, a.a.O. (Fn. 39), S. 194.

78 Vgl. Philip G. Joyce / Robert D. Reischauer, a.a.O. (Fn. 31), S. 96. Diese Argumentation erinnert in ihren Grundzügen an die prärogative Gewalt des Staatsoberhauptes bei John Locke. Eine letztinstanzliche Entscheidungsgewalt dürfe nur zum Wohle des Volkes ausgeübt werden. „For as a good prince, who is mindful of the trust put into his hands and careful of the good of his people, cannot have to much prerogative - that is, power to do good." John Locke, The Second Treatise of Government, London / New York 1970 [1689], \$ 164, S. 201. 
dern zu treten. Der Einsatz oder Nicht-Einsatz der neuen Einflussmöglichkeit dient ihm als Verhandlungsmasse im Vorfeld von Annullierungen. ${ }^{79}$ Dabei vertritt er nicht allein das Gesamtinteresse und nimmt aus diesem Grund Streichungen in den Gesetzesvorlagen vor, sondern "presidents may bargain on behalf of local interests, too "80. Gabel und Hager verweisen darauf, dass der Präsident auch bestrebt ist, Klientelpolitik zu betreiben, insbesondere wenn er wiedergewählt werden möchte: „The president gets elected by winning the electoral votes of states, not necessarily by winning a plurality of support from voters across the nation. In other words, states can be thought of as specific electoral districts to which the president must appeal. Presidents who are seeking reelection or who care whether their party maintains control of the presidency should devote special attention to key states or constituencies." ${ }^{81}$ Der Präsident ist also ebenso wie die Abgeordneten daran interessiert, bei der Wählerschaft für sich zu werben. ${ }^{82}$

Anlässlich der Unterzeichnung des Line-Item Veto Acts im Jahr 1996 betonte der Pressesprecher des Weißen Hauses, dass der Präsident mit der Entscheidung, das selektive Veto einzusetzen, auch Politik in seinem Interesse gestalten wolle. ${ }^{83}$ Anstatt Annullierungen ausschließlich aufgrund eines fiskalischen Gesamtinteresses vorzunehmen, ist es für den Präsidenten ebenso sinnvoll, Abgeordnete mit der Androhung von Streichungen dazu zu bewegen, seinen programmatischen Interessen zu entsprechen ${ }^{84}$, wie Annullierungen außerhalb seiner Agenda vorzunehmen. ${ }^{85}$ Das Line-Item Veto stellt damit einen neuen Einflussraum des Präsidenten dar. Er kann nunmehr legislative Mandate entscheidend beeinflussen, da mit der Bestimmung darüber, wohin welches Geld fließt, Politik tatsächlich gestaltet wird. ${ }^{86}$ Ganz gegen die Absicht der Verfassung steigt der Präsident mit einer Line-Item Veto-Kompetenz zu einem vollwertigen Akteur im Gesetzgebungsprozess auf und bildet eine Art Revisions-, wenn nicht sogar Lenkungsinstanz, die nur dann nicht das letzte Wort spricht, wenn der Kongress die erforderliche Zweidrittelmehrheit zur Ausschaltung aufbringt. „The item veto, rather than acting as a check of wasteful spending, may serve simply to add another member, a rather powerful one, to the logrolling coalition." 87

79 Vgl. Roderick Kiewiet / Mathew D. McCubbins, Appropriations Decisions as a Bilateral Bargaining Game Between President and Congress, in: Legislative Studies Quarterly, 10. Jg. (1985), H. 2, S. 181 - 201; dies., Presidential Influence on Congressional Appropriations Decisions, in: American Journal of Political Science, 32. Jg. (1988), H. 3, S. 713 - 736.

80 Matthew J. Gabel/ Gregory L. Hager, a.a.O. (Fn. 18), S. 22.

81 Ebenda.

82 Vergleiche für neuste Erhebungen zur „Presidential Electoral Connection“ die Studien von Nolan M. McCarty, Presidential Pork: Executive Veto Power and Distributive Politics, in: The American Political Science Review, 94. Jg. (2000), H. 1, S. 117 - 129; Andrew J. Taylor, The Presidential Pork Barrel and the Conditioning Effect of Term, in: Presidential Studies Quarterly, 38. Jg. (2007), H. 1, S. 96 - 109.

83 Vgl. Mike McCurry, White House Press Briefing, 9. April 1996, http://www.presidency.ucsb.edu/ ws/index. php?pid=59378 (Abruf am 16. Mai 2008).

84 Matthew J. Gabel / Gregory L. Hager, a.a.O. (Fn. 18), S. 22.

85 Vgl. Matthew Soberg Shugart / Stephan Haggard, Institutions and Public Policy in Presidential Systems, in: Stephan Haggard / Matthew D. McCubbins (Hrsg.), Presidents, Parliaments, and Policy, Cambridge 2001, S. 64 - 104, S. 101.

86 Vgl. Majid Sattar, a.a.O. (Fn. 11), S. 125.

87 Matthew J. Gabel / Gregory L. Hager, a.a.O. (Fn. 18), S. 22. 


\section{Die Rechtsprechung des US-Supreme Court zum Line-Item Veto Act}

Bei der Verabschiedung des Gesetzes wurde bereits deutlich, dass die unterlegenen Parlamentarier eine verfassungsgerichtliche Prüfung anstreben würden. Einer der einflussreichsten Widersacher des Line-Item Vetos, der Demokratische Senator Robert C. Byrd (West Virginia), beschrieb die Gefahr eines Machtmissbrauchs durch den Präsidenten wie folgt: „This so-called line-item veto act should be more appropriately labeled ,The President Always Wins Bill'. From now on, the heavy hand of the President will be used to slap down Congressional opposition wherever it may exist." 88 Eine erste Appellationsklage unter Führung von Byrd und dem Demokratischen Abgeordneten David E. Skaggs (Colorado) ${ }^{89}$ lehnte der Supreme Court jedoch mit der Begründung ab, dass es sich um eine nicht zulässige abstrakte Normenkontrolle handele, da das Gesetz bis dahin noch nicht angewandt worden war. ${ }^{90}$

Nach erstmaliger Anwendung des Gesetzes im August 1997 stand einer konkreten Normenkontrolle nichts mehr im Wege. In einem ersten Fall monierten die Kläger - die Stadt New York, zwei Krankenhausorganisationen (Greater New York Hospital Association und New York City Health and Hospitals Corporation), ein Krankenhaus (Jamaica Hospital Medical Center) sowie zwei Gewerkschaften des Gesundheitswesens - die Annullierung von Steuerentlastungen und zusätzlicher Etatbereitstellungen aus dem Balanced Budget Act of 1997. ${ }^{91}$ Mit den direkten Zuwendungen hätte die Stadt New York ihren Gesundheits-

88 Robert C. Byrd, Congressional Record - Senate, 27. März 1996, S. S2937.

89 Neben Byrd und Skaggs, zählten die Demokratischen Senatoren Carl Levin (Michigan), Daniel Patrick Moynihan (New York) und der Republikaner Mark Hatfield (Oregon) sowie der Demokratische Abgeordnete Henry Waxman (Kalifornien) zu den Klägern. The Washington Post, Line Item Veto: Raines v. Byrd, http://www.washingtonpost.com/wp-srv/national/longterm/supcourt/ 1996-97/lineitem96.htm (Abruf am 22. Januar 2008); Raines v. Byrd, 26. Juni 1997, http://caselaw.lp.findlaw.com/scripts/getcase.pl?court=US\&navby=case\&vol=000\&invol=96-1671 (Abruf am 22. Januar 2008).

90 Die amerikanische Gerichtsbarkeit unterscheidet zwischen persönlichen (Personal Injury) und verfassungsrechtlichen (Institutional or Official Injury) Schäden. Im Fall Raines v. Byrd war der Supreme Court der Ansicht, dass die Klage der Parlamentarier zulässig gewesen wäre, wenn sie in einem Prozess gegen die Exekutive einen persönlichen - zum Beispiel den Verlust ihres Amtes oder einen konkreten verfassungsrechtlichen Schaden erlitten hätten. In der Sicht des Gerichtes vertraten die Kläger jedoch einen verfassungsrechtlichen Schaden, welcher alle Mitglieder des Kongresses benachteiligen würde (eine Minderung gesetzgebender und politischer Befugnisse) und keinen persönlichen konkreten und bestimmten Schaden. Das Gericht lehnte es in diesem Fall ab, darüber zu entscheiden, ob das Ergebnis ein anderes gewesen wäre, wenn: (1) die Kläger für den Senat und das Repräsentantenhaus in ihrer Gänze zu sprechen autorisiert gewesen wären (tatsächlich lehnten beide Kammern in ihrer Mehrheit den Prozess ab) und (2) es den Parlamentariern an einem Rechtsmittel gegen die Anwendung des Line-Item Vetos vorzugehen, gefehlt hätte. Das Gericht bemerkte, dass die Mitglieder des Kongresses durch die Disapproval Resultion die präsidentiellen Annullierungen von Passagen in Haushaltsgesetzten widerrufen konnten. Vgl. Jay R. Shampansky, Congressional Standing to Sue: An Overview, CRS Report for Congress, RL30280, Washington D.C. 2001; Neal Devins / Michael A. Fitts, The Triumph of Timing: Raines v. Byrd and the Modern Supreme Court's Attempt to Control Constitutional Confrontations, in: Georgetown Law Journal, 86. Jg. (1997), H. 1, S. 351 ff.

91 Vgl. dazu die Benachrichtigung des Präsidenten an den Kongress zu den Streichungen durch den Line-Item Veto Act: 97-3, Cancellation Pursuant to Line Item Veto Act; Balanced Budget Act of 1997, in: Federal Register, Vol. 62, No. 155, 12. August 1997, http://frwebgate.access.gpo.gov/ 
dienst für Bedürftige (Medicaid) aufrechterhalten können. Die unter Zuhilfenahme des Line-Item Vetos gestrichenen Geldzuweisungen hätten ihren Anspruch begründet, Bundesgelder zu erhalten und bestimmte Steuern zu erheben, um ihre Ausgaben auszugleichen. ${ }^{92}$ Nach Aussage des New Yorker Bürgermeisters Rudolph Giuliani hätten die Streichungen 2,6 Milliarden Dollar Mehrkosten für die Stadt verursacht. ${ }^{93}$

Der Agrarverband Snake River Potato Growers aus Idaho klagte in einem zweiten Fall gegen das selektive Veto, das die Abschreibung von Kapitalertragssteuern bei Verkäufen an landwirtschaftliche Genossenschaften wieder aufhob. ${ }^{94}$ Präsident Clinton hatte die Abschreibungen aus dem Taxpayer Relief Act of 1997 annulliert. ${ }^{95}$

Am 12. Februar 1998 gab das Distriktgericht (Federal District Court) in Washington D.C. beiden Klagen statt. Richter Thomas F. Hogans Begründung fiel wie folgt aus: „[The Line-Item Veto Act] improperly allowed the President to pick and choose among portions of an enacted law to determine which ones will remain valid." 96 Auch wenn durch die Streichung - wie bei der Einführung des selektiven Vetos intendiert - öffentliche Gelder hätten eingespart werden können, erklärte Hogan, dass „the Line-Item Veto Act violates the procedural requirements ordained in Article I of the United States Constitution and impermissibly upsets the balance of powers so carefully prescribed by its framers. The Line-Item Veto Act therefore is unconstitutional"97.

Das Weiße Haus beschritt den Instanzenweg. Der Oberste Gerichtshof sollte darüber befinden, ob der Line-Item Veto Act in seiner Ausgestaltung als Enhanced Rescission-Verfahren das Prinzip der Gewaltenteilung verletze. Der Supreme Court veröffentlichte seine Entscheidung gegen das selektive Veto am 25. Juni 1998. Die Verfassungsrichter erklärten mit sechs zu drei Stimmen, dass die Verfassung dem Präsidenten nicht erlaube, einzelne

cgi-bin/getdoc.cgi?dbname=1997_register\&docid=fr12au97-145.pdf (Abruf am 6. Juli 2007). Vgl. auch die durch die Streichungen betroffene Gesetzgebung: H.R. 2015, http://frwebgate.access.gpo.gov/cgi-bin/getdoc.cgi?dbname=105_cong_bills\&docid=f:h2015enr.txt.pdf (Abruf am 6. Juli 2007); P.L. 105-33 http://frwebgate.access.gpo.gov/cgi-bin/getdoc.cgi?dbname=105_ cong_public_laws\&docid=f:publ33.105.pdf (Abruf am 6. Juli 2007); H.Rept. 105-217, http:// frwebgate.access.gpo.gov/cgi-bin/getdoc.cgi?dbname=105_cong_reports\&docid=f:hr217.pdf (Abruf am 6. Juli 2007).

92 Vgl. The New York Times, Challenging the Line Item Veto, 18. Oktober 1997, S. 14.

93 Vgl. Robert Pear, U.S. Judge Rules Line Item Veto Act Unconstitutional, in: The New York Times vom 13. Februar 1998, S. 1.

94 Vgl. Linda Greenhouse, Justices to Hear President's Appeal on Line Item Veto Ruling, in: The New York Times vom 28. Februar 1998, S. 7; Robert Pear, Justice Dept. Belatedly Finds New Defense of Line Item Veto, in: The New York Times vom 26. März 1998, S. 18.

95 Vgl. 97-1, Cancellation Pursuant to Line Item Veto Act, Taxpayer Relief Act of 1997, in: Federal Register, Vol. 62, No. 155, 12. August 1997, http://frwebgate.access.gpo.gov/cgi-bin/getdoc. cgi?dbname=1997_register\&docid=fr12au97-146.pdf (Abruf am 6. Juli 2007); 97-2, Cancellation Pursuant to Line Item Veto Act, Taxpayer Relief Act of 1997, in: Federal Register, Vol. 62, No. 155, 12. August 1997; H.R. 2014, http://frwebgate.access.gpo.gov/cgi-bin/getdoc.cgi? dbname=105_cong_bills\&docid=f:h2014enr.txt.pdf (Abruf am 6. Juli 2007); P.L. 105-34 http:// frwebgate.access.gpo.gov/cgi-bin/getdoc.cgi?dbname=105_cong_public_laws\&docid =f:publ34.105.pdf (Abruf am 6. Juli 2007); H.Rept. 105-220, http://frwebgate.access.gpo.gov/ cgi-bin/getdoc.cgi?dbname=105_cong_reports\&docid=f:hr220.pdf (Abruf am 6. Juli 2007).

96 Robert Pear, a.a.O. (Fn. 93).

97 Thomas F. Hogan, Quotation of the Day, in: The New York Times vom 13. Februar 1998, S. 1; The New York Times, Excerpts From Decision Against the Line Item Veto, 13. Februar 1998, S. 23. 
Passagen aus Haushaltsgesetzen zu streichen. Die Essenz des Urteils liegt in der Beurteilung von Richter Paul Stevens (Majority Opinion): „[T] he line-item veto law violates a constitutional requirement that legislation be passed by both houses of Congress and presented in its entirety to the president for signature or veto." ${ }^{98}$ Danach bleibe dem Präsidenten allein ein Entweder-Oder. ${ }^{99}$ Ein Herumstreichen im Gesetzestext werde von der Verfassung nicht gedeckt. ${ }^{100}$ Richter Stevens begründete weiter, „this act gives the President the unilateral power to change the text of duly enacted statutes“ und sei insofern ein „functional equivalent of partial repeals of acts of Congress" ${ }^{101}$. Ein Line-Item Veto sei nicht in der Verfassung vorgesehen.

Drei Richter wichen von der Mehrheitsmeinung ab (Dissenting Opinion): „There is not a dime's worth of difference between Congress's authorizing the president to cancel a spending item, and Congress's authorizing money to be spent on a particular item at the president's discretion" 102 , schrieb Antonin Scalia, der bis dahin als strenger Hüter der formalistischen Gewaltenteilungsdoktrin (Strict Constructionist) in Erscheinung getreten war. ${ }^{103}$

In seinem Urteil erklärte der Oberste Gerichtshof das Line-Item Veto für verfassungswidrig. Für ihn stellten die Streichungen schwerwiegende Eingriffe in eine Gesetzesvorlage dar. Während über die Ursprungsvorlage in beiden Häusern des Kongresses abgestimmt wurde, fand gleiches nicht bei dem vom Präsidenten geänderten und in Kraft getretenen Gesetz statt. Oder anders formuliert: Der Kongress ist der Gesetzgeber, das vom Präsidenten veränderte Gesetz ist so nicht vom Kongress verabschiedet worden. Dies, so das Gericht, verletze die Gewaltenteilungsdoktrin der Verfassung. ${ }^{104}$ Der Demokratische Abgeordnete James A. McDermott (Washington) bringt es auf den Punkt: „Do you understand the separation of powers? We are the ones who decide how the money gets spent, not the President." 105

\section{Line-Item Veto: Gestärkte Position des Präsidenten in der legislativen Arena}

Zu Beginn seiner zweiten Amtszeit übte Präsident Clinton das Line-Item Veto erstmals am 11. August 1997 und bis zum Urteilsspruch des Supreme Courts am 25. Juni 1998 regelmäßig aus. Er nahm in elf Gesetzen insgesamt 82 Annullierungen vor. Clinton nutzte das selektive Veto aus programmatischen und parteipolitischen Motiven. Zur intendierten Haushaltskonsolidierung trug es jedoch nicht bei. Viel bedeutender sind die Veränderungen der Machtbalance zwischen Präsident und Kongress, die mit dem Line-Item Veto einhergehen - auch wenn Wandlungen des Legislativ-Exekutiv-Verhältnisses schon in der Ver-

98 Paul Stevens, Opinion of the Court, in: William J. Clinton v. City of New York, a.a.O. (Fn 10).

99 Vgl. Majid Sattar, a.a.O. (Fn. 11), S. 36.

100 Vgl. Helen Dewar / Joan Biskupic, Courts Strikes Down Line-Item Veto, in: The Washington Post vom 26. Juni 1998, S. 1.

101 Paul Stevens, a.a.O. (Fn. 98).

102 Antonin Scalia, Opinion of Justice Scalia, in: William J. Clinton v. City of New York, a.a.O. (Fn. 10).

103 Vgl. Birgit Oldopp, a.a.O. (Fn. 63), S. 109.

104 Vgl. ebenda, S. 70.

105 James A. McDermott, Congressional Record - House, 20. Juni 2006, S. H4226. 
fassung der USA angelegt und daher an sich kein neues Problem sind: „The balance between President and Congress [has] gone through nearly two centuries of ups and downs. "106 Das jeweilige Machtverhältnis hängt von unterschiedlichen Faktoren ab. Zu ihnen gehören externe, gesellschaftliche ebenso wie solche, die sich auf die internen Akteure Kongress und Präsident beziehen. Obwohl Gewaltenteilungstheoretiker nach einem Optimum der Machtverteilung, nach einem institutionellen Gleichgewicht suchen ${ }^{107}$, scheint die Dynamik vielmehr funktional zu sein: „Our proper objective is neither a dominant presidency nor an aggressive Congress but, within the strict limits of what the constitution mandates, a shift of emphasis according to the needs of the time and the requirements of public policy. "108 Die Verfassung erlaubt aber nicht, dass der Präsident Gesetze einseitig verändert oder aufhebt. Die haushaltspolitische Autorität liegt bei der Legislative und kann nicht teilweise an die Exekutive abgegeben werden - eine herausgehobene Stellung des Präsidenten im Haushaltsprozess ist nicht vorgesehen. Mit dem selektiven Veto hatte der Kongress dem Präsidenten jedoch genau diese übertragen. ${ }^{109}$

In einer der letzten grundlegenden wissenschaftlichen Betrachtungen zum US-amerikanischen Parlamentarismus in deutscher Sprache beschreibt zum Ende der Präsidentschaft Ronald Reagans Uwe Thaysen in seinem Resümee den Trend „zurück zum ,congressional government" "110. Für ihn rechtfertigten die Entwicklungen im amerikanischen Regierungssystem der 1980er Jahre sogar die Prognose einer „auf längere Zeit anhaltenden Selbstbehauptung eines gegenüber der Exekutive deutlich erstarkten Kongresses "111. Patrick Horst bescheinigt dieser Prognose eine bemerkenswerte Nachhaltigkeit: „Die ,Selbstbehauptung' des Kongresses gegenüber den ersten beiden Nachfolgern Reagans im Amt des Präsidenten, George H. W. Bush und William J. Clinton, steht nicht in Frage; diagnostiziert oder beklagt wurde vielmehr auch in der Nach-Reagan-Ära wie eh und je die Ohnmacht des Präsidenten vis-à-vis dem Kongress. " 112 Es erscheint fraglich, ob dieser Befund auch für den 105. Kongress der Jahre 1997/1998, in dem Präsident Clinton über die zusätzliche Machtbefugnis eines selektiven Vetos verfügte, aufrecht erhalten werden kann. Die herausgehobene Stellung des Kongresses trifft für die von Thaysen und Horst gewählten Kriterien und im Gesamtbild der Legislativ-Exekutiv-Beziehungen sicherlich nach wie vor zu. Jedoch muss diese Einschätzung für die Periode des Line-Item Vetos zumindest hinsichtlich des Haushaltsverfahrens modifiziert werden. Die Art und Weise, in der Clinton das Line-Item Veto nutzte, lässt auf den Gebrauch zukünftiger Präsidenten schließen. In dieser Hinsicht sind die jüngsten Bestrebungen zur Wiedereinführung des Line-Item Vetos zu überdenken.

106 James L. Sundquist, The Decline and Resurgence of Congress, Washington D.C. 1981, S. 461.

107 Vgl. Charles O. Jones, Separate but Equal. Congress and the Presidency, Chatham 1995, S. 20 f.

108 Senator J. William Fulbright zitiert nach ebenda, S. 20.

109 Vgl. Calvin Bellamy, Item Veto: Dangerous Constitutional Tinkering, in: Public Administration Review, 49. Jg. (1989), H. 1, S. $46-51$.

110 Uwe Thaysen / Roger H. Davidson / Robert G. Livingston, US-Kongress und Deutscher Bundestag im Vergleich. Ein ergänzendes Resümee, in: dies. (Hrsg.), US-Kongress und Deutscher Bundestag. Bestandsaufnahmen im Vergleich, Opladen 1988, S. 520.

111 Ebenda, S. 547.

112 Patrick Horst, a.a.O. (Fn. 27), S. 680. 